\title{
FRACTIONAL LOCATION PROBLEMS
}

\author{
A. I. BARROS* \\ TNO Physics and Electronics Laboratory P.O. Box g6264, 2509 JG, THe Hague, The Netherlands
}

\author{
J. B. G. FRENK
}

Erasmus University Rotterdam, P.O. Box 1738, 3000 DR Rotterdam, the Netherlands

and

\section{J. GROMICHO}

ORTEC Consultants bv, Groningenweg 6-33, 2803 PV Gouda, the Netherlands

\begin{abstract}
In this paper we analyze some variants of the classical uncapacitated facility location problem with a ratio as an objective function. Using basic concepts and results of fractional programming, we identify a class of one-level fractional location problems which can be solved in polynomial time in terms of the size of the problem. We also consider the fractional two-echelon location problem, which is a special case of the general two-level fractional location problem. For this two-level fractional location problem we identify cases for which its solution involves decomposing the problem into several one-level fractional location problems. (C) 1997 Elsevier Science Ltd. All rights reserved
\end{abstract}

Key words: discrete location, fractional programming.

\section{INTRODUCTION}

Given some population of clients, discrete location deals with the problem of deciding where to locate facilities among a finite set, in such a way that a certain criterion is optimized. Traditionally, the criterion is to maximize the total net profit, which measures the gains of a certain location decision, i.e. the difference between the sum of the profits of serving each client via certain facilities and the fixed costs associated with those facilities. However, as Barros (1995), Revelle and Laporte (1993), and Hansen et al. (1994) show, it might also be important in some economical applications to consider the criterion of maximizing the profitability index, i.e. maximizing the ratio between the gains and the fixed costs. Solving these discrete location problems where a ratio of two functions has to be optimized requires not only the use of classical integer programming techniques but also of fractional programming techniques. At first sight, this may appear to complicate the already difficult location problems, but as we will see, it can also simplify the solution procedure dramatically.

In this paper, some variants of the uncapacitated facility location problem with a ratio as an objective function are discussed. Using basic concepts and results of fractional programming, it is possible to identify one-level fractional location problems which can be solved in

*This research was carried out at the Econometric Institute, Erasmus University Rotterdam, the Netherlands and was supported by the Tinbergen Institute Rotterdam. 
polynomial time in terms of the size of the problem. The solution procedure encountered is based on the characterization of the optimal solution of the linear version of these problems when its optimal objective value is known to be equal to zero. Using the same reasoning, we can extend this result for a special fractional two-level location problem and show that for this case the problem can be decomposed into as many one-level fractional location problems as the number of facilities.

This paper is organized as follows. We begin by providing a small overview of the basic results of fractional programming. In Section 3 we analyze some fractional one-level location problems, while in Section 4 we discuss the special case of the fractional two-echelon location problem.

Throughout the paper we will denote the optimal objective value of an optimization problem $(P)$ by $\vartheta(P)$. We will also say that the optimization problem $(P)$ is finitely solvable if $\vartheta(P)$ is finite and there exists some feasible solution that attains the optimal objective value.

\section{FRACTIONAL PROGRAMMING}

To introduce fractional programming problems (see Schaible (1995) for an updated survey) let $\chi \subseteq \mathbb{R}^{n}$ be a set and $\mathrm{f}, g: \chi \rightarrow \mathbb{R}$ some finite valued functions with $g(x)>0$ for every $x \in \chi$. A fractional programming problem $(P)$ is given by

$$
\sup _{x \in \chi} \frac{f(x)}{g(x)}
$$

Observe that the above problem also covers combinatorial fractional programs, since $\chi$ can also represent a finite subset of $\mathbb{R}^{\prime \prime}$; see Hashizume et al. (1987). In the remainder, we will always assume that $(P)$ is finitely solvable.

One of the most popular approaches to solving $(P)$ is the parametric approach (see Schaible, 1995), which consists of analyzing the class of optimization problems given by

$$
F(\lambda):=\sup _{x \in \chi}\{\mathbf{f}(\mathbf{x})-\lambda g(\mathbf{x})\}
$$

with $\lambda \in \mathbb{R}$. It is always assumed that $\left(P_{\lambda}\right)$ is finite solvable. We can now state the relation between the original problem $(P)$ and its associated parametric problem $\left(P_{\lambda}\right)$. The proof of this result can be found in Dinkelbach (1967). Keep in mind we always assume that $(P)$ and $\left(P_{\lambda}\right)$ for every $\lambda \in \mathbb{R}$ are finitely solvable. This assumption holds for the location problems considered in this paper.

\section{Lemma 2.1.}

- (a) The function $F: \mathbb{R} \rightarrow \mathbb{R}$ is convex, continuous and strictly decreasing.

- (b) If $\lambda_{*}$ denotes the optimal objective value of $(P)$ then $F\left(\lambda_{*}\right)=0$.

- (c) $F(\lambda)=0$ implies $\lambda=\lambda_{*}$.

- (d) The optimal solution set of $\left(\mathrm{P}_{\lambda_{*}}\right)$ equals the optimal solution set of $(P)$.

From Lemma 2.1, it follows that solving $(P)$ is equivalent to finding the unique root of the nonlinear univariate equation $F(\lambda)=0$. Thus, computing the root of $F(\lambda)=0$ can be done by 
usual numerical methods, like Newton's method or the bisection method. Actually, one of the most popular methods to find the root of this equation is an extension of Newton's method, usually known as Dinkelbach's algorithm; see Dinkelbach (1967).

This strategy seems appropriate for combinatorial fractional programs, since the resulting parametric problem corresponds to a combinatorial problem of a similar nature. It is important to stress that within the combinatorial fractional programming class, there exist some problems that are quite easily solvable. In fact, if a problem consisting of optimizing a linear type of objective function on a certain feasible set can be solved in polynomial time then its fractional counterpart can also be solved in polynomial time as shown by Meggido (1979). As we will see in Section 3, our results are of a somehow reciprocal nature. In fact we show that there exist some classes of location problems inherently difficult, which have easy fractional counterparts.

\section{ONE-LEVEL FRACTIONAL LOCATION PROBLEMS}

We will start our analysis of the one-level fractional location problems by discussing the basic uncapacitated facility location problem; see Cornuéjols et al. (1990) for an excellent survey. This problem consists of choosing where to locate facilities, selected from a discrete set of possible locations, in order to maximize the profit associated with satisfying the demand of a given set of clients. Usually, setting up a facility involves significant costs that are not proportional to the production level of this facility, and which depend on its location. Hence, fixed costs of opening a given facility depending on the location are considered. Facilities are assumed to have unlimited capacity, i.e. any facility can satisfy the demand of all clients. The problem can now be formalized as follows. Let $I:=\{1, \ldots, m\}$ denote the set of clients and $J:=\{1, \ldots, p\}$ the set of sites where facilities can be located. Let also $f_{j}$ denote the fixed cost of opening facility $j$, and $c_{i j}$ the profit associated with satisfying the demand of client $i$ from facility $j$. Using the terminology in location problems, a facility $j$ is "open" when that facility is established in location $j$ and so, if

$$
y_{j}= \begin{cases}1 & \text { if facility } j \text { is open } \\ 0 & \text { otherwise }\end{cases}
$$

and $x_{i j}$ denotes the fraction of the demand of client $i$ served by facility $j$, we have the following formulation of the uncapacitated facility location problem

$$
\begin{gathered}
\max \sum_{i \in I} \sum_{j \in J} c_{i, j} x_{i j}-\sum_{j \in J} f_{j} y_{j} \\
\text { s.t.: } \sum_{j \in J} x_{i, j}=1 \forall i \in I \\
x_{i j} \leq y_{j} \forall i \in I, j \in J \\
y_{j} \in\{0,1\} \forall j \in J \\
x_{i j} \geq 0 \forall i \in I, j \in J
\end{gathered}
$$

On the other hand, if the criteria to be optimized is now the profitability index, the above linear objective function is replaced by 


$$
\max \frac{\sum_{i \in I} \sum_{j \in J} c_{i j} x_{i j}}{\sum_{j \in J} f_{j} y_{j}}
$$

In order to have a well defined fractional program, we assume that the fixed costs $f_{j}$ are positive for every $j$. Due to the combinatorial nature of the above integer fractional programming problem, it seems quite reasonable to apply a parametric approach to solve (FFL). Therefore, consider the associated parametric problem,

$$
\begin{gathered}
\max \sum_{i \in,} \sum_{j \in J} c_{i j} x_{i j}-\lambda \sum_{j \in J} f_{j} y_{j} \\
\text { s.t.: (1),(2),(3),(4) }
\end{gathered}
$$

The combinatorial structure of the above problem immediately suggests the use of a parametric approach, although the associated parametric problem is an NP-hard problem; see Cornuéjols et al. (1990). On the other hand, from Lemma 2.1 it follows that for $\lambda$ equal to $\vartheta(F F L)$ the value of the associated parametric problem is zero. Hence, we will investigate the class of uncapacitated facility location problems that have zero as the optimal objective value.

Lemma 3.1. If $c_{i j} \geq 0$ for all $i \in I, j \in J, f_{j} \geq 0$ for all $j \in J$ and the optimal objective value of (FL) equals zero, then there exists an optimal solution to this problem with only one facility open.

Proof. Let $(\boldsymbol{y}, \boldsymbol{x})$ be a optimal solution of the problem $(F L)$ satisfying $9(F L)=0$. Notice that this solution is different from the null vector and denote the set of open facilities by $J^{*}$. By assumption we have

$$
0=9(F L)=\sum_{j \in J^{*}}\left(\sum_{i \in,} c_{i, j} x_{i j}-f_{j}\right)
$$

Let $j^{*} \in \mathrm{J}^{*}$. If $\Sigma_{i \in l}, c_{\mathrm{ij}^{*}} \mathrm{x}_{\mathrm{ij}} \mathrm{j}^{*}-\mathrm{f}_{\mathrm{j}^{*}}>0$ then by the nonnegativity assumption on $c_{i j}$ we also have that $\Sigma_{i \in I} c_{i j^{*}}-f_{j^{*}} \geq \Sigma_{i \in I}, c_{i j *} x_{i j^{*}}-f_{j^{*}}>0$. This implies that the solution $\left(\boldsymbol{y}^{\prime}, \boldsymbol{x}^{\prime}\right)$ given by

$$
y_{j}{ }^{\prime}=\left\{\begin{array}{ll}
1 & \text { if } j=j^{*} \\
0 & \text { otherwise }
\end{array} \text { and } \quad x_{i j}{ }^{\prime}= \begin{cases}1 & \text { for all } i \in I \text { and } j=j^{*} \\
0 & \text { otherwise }\end{cases}\right.
$$

is feasible with a positive objective value. Since $9(F L)=0$, this contradicts the optimality of $(\boldsymbol{y}, \boldsymbol{x})$ and so for $j^{*} \in J^{*}$ we must have according to (5) that $\Sigma_{i \in l} c_{i j} * x_{i j^{*}}-f_{j^{*}}=0$. This implies that $\Sigma_{i \in l} c_{i j^{*}}-f_{j^{*}} \geq \Sigma_{i \in 1} c_{i j *} x_{i j^{*}}-f_{j^{*}}=0$ and since the solution given by (6) is feasible it follows that $\Sigma_{i \in I}, c_{i j^{*}}-f_{j^{*}} \leq 9(F L)=0$. Hence, $\Sigma_{i \in I} c_{i j^{*}}-f_{j^{*}}=0$ and so (6) solves $(F L)$ which concludes the proof.

Using the above Lemma 3.1 it is possible to characterize an optimal solution of (FFL)

Proposition 3.2 If $c_{i j} \geq \mathbf{0}$ for all $i \in I, j \in J, f_{j}>0$ for all $j \in J$ then there exists an optimal solution of (FFL) where only one facility is open. Moreover,

$$
\vartheta(\mathrm{FFL})=\max _{j \in J} \frac{\sum_{i \in,} c_{i j}}{\mathrm{f}_{j}}
$$


Proof. From Lemma 2.1 it follows that the associated parametric problem has optimal objective value equal to zero for $\lambda_{*}=\vartheta(F F L)$. Hence, by Lemma 3.1 (with $f_{j}$ replaced by $\lambda_{*} f_{j}$ ) we can find an optimal solution of $\left(\mathrm{FFL}_{\lambda_{2}}\right.$ ) with only one facility open and so again by Lemma 2.1 the first part is proved. The second part follows now easily.

Using the above proposition we determine the following result.

Theorem 3.3. If $c_{i j} \geq 0$ for all $i \in I, j \in J, f_{j}>0$ for all $j \in J$ then $(F F L)$ can be solved in $0(p m)$ time.

Whenever the assumption on the profitability associated with the clients, i.e. $c_{i j} \geq 0$ for all $i \in I, j \in J$ cannot be ensured, then $(F F L)$ may have only optimal solutions with more than one facility open. To show this, consider the following example.

Example 3.4. Let $I=\{1,2\}$ denote the set of clients, $J=\{1,2\}$ the set of facilities and consider the following parameters

\begin{tabular}{l|rr}
$c_{i j}$ & 1 & 2 \\
\hline 1 & 20 & -5 \\
2 & -5 & 20
\end{tabular}

Let also the fixed costs of both facilities be equal to 5.

In this case opening only one facility yields a profitability index of 3 , while opening two facilities yields the bigger profitability index 4 .

There exist some additional modifications of the pure fractional uncapacitated facility location problem $(F F L)$ that transform this problem into one for which Proposition 3.2 does not hold. For instance, whenever it is assumed that the investment is composed not only of the fixed costs of opening facilities, but also of an additional initial fixed investment $c>0$. The model $(F F L)$ will then have the following objective function

$$
\max \frac{\sum_{i \in I} \sum_{j \in J} c_{i j} x_{i j}}{\sum_{j \in J} f_{j} y_{j}+c}
$$

In this case it is not possible to guarantee, even in the case that all $c_{i j} \geq 0$, that there exists one optimal solution of $(F F L c)$ which has only one facility open. In fact, Example 3.4 with $c_{12}=c_{21}=5$ and initial investment $c=10$ shows that the only optimal solution is given with both facilities open.

Another type of investment that may be considered in location theory corresponds to the so-called expansion costs of manufacturing. The expansion cost at facility $j, e_{j}$, associated with client $i$ can be viewed as the costs of purchasing the machinery necessary to produce the demand $d_{i}$ of client $i$ at site $j$. In this case the total investment cost corresponds to the sum of the usual fixed costs $\Sigma_{j \in J} f_{j}$ with $\Sigma_{i \in I} \Sigma_{j \in J} e_{j} d_{i} x_{i j}$. In this case the denominator of the fractional objective function associated with this variant is given by:

$$
\max \frac{\sum_{i \in I} \sum_{j \in J} c_{i j} x_{i j}}{\sum_{j \in J} f_{j} y_{j}+\sum_{i \in I} \sum_{j \in J} e_{j} d_{i} x_{i j}}
$$


Unfortunately, in this case the results derived so far are no longer valid as the next example shows.

Example 3.5. As before, let $I=\{1,2\}$ denote the set of clients, $J=\{1,2\}$ the set of facilities and consider the following parameters

\begin{tabular}{l|rr}
$c_{i j}$ & 1 & 2 \\
\hline 1 & 20 & 5 \\
2 & 5 & 20
\end{tabular}

\begin{tabular}{l|rr}
$e_{j} d_{i}$ & 1 & 2 \\
\hline 1 & 5 & 5 \\
2 & 10 & 10
\end{tabular}

Let also the fixed costs of both facilities be equal to 5 .

Observe that in this case, the only optimal solution is given by opening both facilities.

Another variant of this basic model is given by the case where the location of the facilities has to be decided not according to pre-determined clients, but to potential clients; see Revelle and Laporte (1993). This model is suitable for those practical situations, where no pre-established contracts with clients exist and therefore, there is not any obligation of satisfying the demand of the clients. In fact, as mentioned by Revelle and Laporte (1993), it is important in this case to maximize the ratio between the profits and the associated investments. The profits associated with serving client $i$ via facility $j$ are determined by a function of the production costs at facility $j$, the demand $d_{i}$ of client $i$, the selling price of client $i$ and the transportation costs between client $i$ and facility $j$. In this model, it is also assumed that there exist expansion costs as defined above, and so it is given by (see Revelle and Laporte (1993))

$$
\begin{gathered}
\max \frac{\sum_{i \in I} \sum_{j \in J} c_{i j} x_{i j}}{\sum_{\in J} f_{j} y_{j}+\sum_{\in I} \sum_{j \in J} e_{i} d_{i} x_{i j}} \\
\text { s.t.: } \sum_{j \in J} x_{i j} \leq 1 \forall i \in I
\end{gathered}
$$

$$
\text { (2),(3),(4) }
$$

Observe that, if for the classical uncapacitated facility location problem there would exist a facility $j$ such that $f_{j}=0$ and $c_{i j}=0$ for all $i \in I$ then $(F F L)$ could be simplified by removing this facility and replacing constraint (1) by (7). This simplified problem reduces to the optimization problem $(F R F L)$ with no expansion costs.

Notice that $(F R F L)$ does not correspond to a well defined problem since at the feasible solution $(0, \ldots, 0)$ the numerator and denominator of the objective function equal zero. However, from an investor's point of view such a solution is not interesting since it corresponds to not investing at all. Moreover, the profitability index is used as an economical 
criterion whenever there are diverse investment options; see Brealey and Myers (1988). Hence, it is essential to check a priori if not investing is the only optimal solution. Before continuing this analysis, we need to assume that in $(F R F L)$ the fixed costs $f_{j}$ are positive and the demand $d_{i}$ of client $i$ as well as the expansion costs $e_{j}$ of a facility are nonnegative. Clearly, if it is decided that an investment is made and hence facilities will be opened, our optimization problem has the form

$$
\begin{gathered}
\max \frac{\sum_{i \in I} \sum_{j \in J} c_{i j} x_{i, j}}{\sum_{j \in J} f_{.} y_{j}+\sum_{i \in I} \sum_{j \in J} e_{j} d_{i} x_{i j}} \\
\text { s.t.: } \sum_{j \in J} y_{j} \geq 1
\end{gathered}
$$

$$
(7),(2),(3),(4)
$$

and this corresponds to a well defined fractional programming problem. Clearly, if all parameters $c_{i j}$ in (FRFLO) are nonpositive, an optimal solution would be to serve none of the clients, i.e. $x_{i j}=0$ for every $i$ and $j$, and so our initial decision to invest in this project would be irrational. Therefore we assume that at least one of the parameters $c_{i j}$ is positive and this yields that the optimal objective value of (FRFLO) is positive. To characterize an optimal solution of $(F R F L O)$ we start as before with an analysis of the associated parametric problem

$$
\begin{gathered}
F(\lambda):=\max \sum_{i \in I} \sum_{j \in J} c_{i, j} x_{i j}-\lambda\left(\sum_{j \in J} f_{j} y_{j}+\sum_{i \in I} \sum_{j \in J} e_{j} d_{i} x_{i j}\right) \\
\text { s.t.: (8),(7),(2),(3),(4) }
\end{gathered}
$$

The above parametric problem is related to the following uncapacitated facility location problem where the assignment constraints (1) are replaced by (7), and (8) is added

$$
\begin{array}{r}
\max \sum_{i \in I} \sum_{j \in J} c_{i j} x_{i j}-\sum_{j \in J} f_{, j} y_{j} \\
\text { s.t.: (8),(7),(2),(3),(4) }
\end{array}
$$

A similar result, as obtained in Lemma 3.1, can now be derived for (RFLO). Observe that in the next lemma no assumptions about the coefficients $c_{i j}$ and $f_{j}, i \in I, j \in J$ are required.

Lemma 3.6. If the optimal objective value of (RFLO) equals zero, then there exists an optimal solution of $(R F I . O)$ with only one facility open.

Proof. Let $(y, x)$ be an optimal solution of $(R F L O)$, with the set of open facilities given by $J^{*} \neq \varnothing$. By assumption we have that

$$
0=9(R F L O)=\sum_{j \in J^{*}}\left(\sum_{i \in I} c_{i j} x_{i j}-f_{j}\right)
$$


Let $j^{*} \in J^{*}$. If $\Sigma_{i \in I} c_{i j j^{*}} x_{i j^{*}}-\mathrm{f}_{j^{*}}>0$ then the solution $\left(y^{\prime}, x^{\prime}\right)$ given by

$$
y_{j}^{\prime}=\left\{\begin{array}{ll}
1 & \text { if } j=j^{*} \\
0 & \text { otherwise }
\end{array} \text { and } x_{i j}{ }^{\prime}= \begin{cases}x_{i j^{*}} & \text { for all } i \in I, j=j^{*} \\
0 & \text { otherwise }\end{cases}\right.
$$

is feasible with a positive objective value and this contradicts $9(R F L O)=0$. Hence, for every $j^{*} \in J^{*}$ it follows that $\Sigma_{i \in I} c_{i j^{*}} x_{i j^{*}}-f_{j^{*}} \leq 0$ and due to (9) we have $\Sigma_{i \in I} c_{i j^{*}} x_{i j^{*}}-f_{j^{*}}=0$ for every $j^{*} \in J^{*}$. Therefore, for any $j^{*} \in J^{*}$, the solution given by (10) solves $(R F L O)$.

Using Lemma 3.6 it is possible to characterize an optimal solution of (FRFLO).

Proposition 3.7. If at least one of the parameters $c_{i j}$ is positive and $f_{j}>0$, for all $j \in J$, then there exists an optimal solution of (FRFLO), also of (FRFL), with only one facility open. Moreover,

$$
\vartheta(F R F L O)=\max _{j \in J}\left\{\max \left\{\frac{\sum_{i \in I} c_{i, j} x_{i, j}}{f_{j}+\sum_{i \in I} e_{j} d_{i} x_{i j}}: 0 \leq x_{i, j} \leq 1, \text { for all } i \in I\right\}\right\}
$$

Proof. From Lemma 2.1 it follows that the associated parametric problem $\left(F R F L O_{\lambda}\right.$ ) has an optimal objective value equal to zero for $\lambda_{*}=9(F R F L O)>0$ and there exists a nonzero optimal solution of this parametric problem. Hence, by Lemma 3.6 (with $f_{j}$ replaced by $\lambda * f_{j}$ and $c_{i j}$ by $\left.c_{i j}-\lambda_{*} e_{j} d_{i}\right)$ we can find an optimal solution of $\left(\mathrm{FRFLO}_{\lambda}\right)$ with only one facility open. Applying Lemma 2.1 again yields the existence of an optimal solution of (FRFLO) with only one facility open, and hence the first result is proved. Using this result relation (11) follows trivially.

Observe that in order to determine the optimal objective value of (FRFLO), $(F R F L)$ in the nontrivial case, i.e. if at least one $c_{i j}$ is positive, we must compute (11), which corresponds to solving $p$ simple allocation problems. Moreover, each of these allocation problems equals a linear fractional programming problem of the form

$$
\max _{0 \leq x_{i} \leq 1} \frac{\sum_{i \in I} a_{i} x_{i}}{\beta+\sum_{i \subset 1} b_{i} x_{i}}
$$

The above problem can be solved in $0(m)$ using the algorithm described in Hansen et al. (1991).

Theorem 3.8. If at least one parameter $c_{i j}$ is positive and $f_{j}>0$ for all $j \in J$, the fractional location problem (FRFLO) can be solved in polynomial time with a complexity order of at most $0(p m)$

The model proposed by Revelle and Laporte (1993) assumes the existence of expansion costs. Clearly, if these costs are zero the above results are still valid. Moreover, in this case the allocation problem (12) reduces to a trivial linear programming problem.

We will conclude this section by remarking that, similarly to what happened for the ( $F F L)$, there are some generalizations of this model that compromise the above results. As an 
example, we mention the existence of an additional initial fixed investment $c>0$. In this case the objective function is replaced by

$$
\max \frac{\sum_{i \in I} \sum_{j \in J} c_{i j} x_{i j}}{c+\sum_{j \in J} f_{j} y_{j}+\sum_{i \in I} \sum_{j \in J} e_{j} d_{i} x_{i j}}
$$

In fact, considering the initial investment $c=10$ in Example 3.5, the only optimal solution is given by opening both facilities.

\section{TWO-LEVEL FRACTIONAL LOCATION PROBLEMS}

So far we have only considered one-level fractional location problems. We will consider now a special case of the general two-level facility location problem (see Barros and Labbé (1994)) and the so-called two-echelon location problem (Gao and Robinson (1992)). This two-level model assumes that for a given set of clients $I=\{1, \ldots, m\}$ requiring the services of a pair (facility, depot) one has to decide where to locate both the facilities among a finite set of sites $J=\{1, \ldots, p\}$ and where to locate the depots among a finite set of sites $K=\{1, \ldots, q\}$. The profits associated with satisfying the demand of client $i$ via facility $j$ and depot $k$ are represented in this model by $c_{i j k}$ and there exist some fixed costs to be taken into consideration: the fixed cost of opening facility $j$ is $f_{j}$ and the fixed cost of having facility $j$ and depot $k$ operating together equals $F_{j k}$. Clearly, a linear type of optimization criteria consists of determining the location of the pairs of facilities and depots in order to maximize the sum of the profits of serving each client from exactly one facility and one depot minus the fixed costs of opening those facilities and also the fixed costs of having facilities and depots operating together. If

$$
\begin{aligned}
& y_{j}= \begin{cases}1 & \text { if facility } j \text { is opencd } \\
0 & \text { otherwise }\end{cases} \\
& \mathrm{t}_{\mathrm{jk}}= \begin{cases}1 & \text { if } j \text { and } k \text { are operating together } \\
0 & \text { otherwise }\end{cases}
\end{aligned}
$$

and $x_{i j k}$ denotes the fraction of the demand of client $i$ served by the operating pair $(j, k)$ then this yields the following formulation

$$
\begin{gathered}
\max \sum_{i \in I} \sum_{j \in J} \sum_{k \in K} c_{i j k} x_{i j k}-\sum_{j \in J} f_{j} y_{j}-\sum_{j \in J} \sum_{k \in K} F_{j k} t_{j k} \\
\text { s.t.: } \sum_{j \in J} \sum_{k \in K} x_{i j k}=1 \forall i \in I \\
x_{i j k} \leq t_{j k} \forall i \in I, j \in J, k \in K \\
t_{j k} \leq y_{j} \forall j \in J, k \in K \\
y_{j,} t_{j k} \in\{0,1\} \forall j \in J, k \in K \\
x_{i j k} \geq 0 \forall i \in I, j \in J, k \in K
\end{gathered}
$$

On the other hand, the corresponding fractional problem is given by 


$$
\begin{aligned}
\max \frac{\sum_{i \in I} \sum_{j \in J} \sum_{k \in K} c_{i j k} x_{i j k}}{\sum_{j \in J} f_{j} y_{j}+\sum_{j \in J} \sum_{k \in K} F_{j k} t_{j k}} \\
\text { s.t.: (13),(14),(15),(16),(17) }
\end{aligned}
$$

We will also assume that $f_{j}>0, j \in J$, and $F_{j k}>0, j \in J, k \in K$. A similar result as obtained in Lemma 3.1 can now be derived.

Lemma 4.1. If $c_{i j k} \geq 0$ for all $i \in I, j \in J, k \in K$, and the optimal objective value of the (2EL) equals zero, then there exists an optimal solution to this problem with only one facility open.

Proof. Let $(t, y, x)$ be an optimal solution of the $(2 E L)$. Notice that this solution is different from the null vector and denote the set of operating pairs by $T^{*}$. Let also $J^{*}$ define the set of open facilities and $K_{j}$ represent the set of depots operating with facility $j \in J^{*}$. By assumption, we have

$$
0=\vartheta(2 E L)=\sum_{j \in J^{*}}\left(\sum_{k \in K_{j}}\left(\sum_{i \in I} c_{i, j k} x_{i, j k}-F_{j k}\right)-f_{j}\right)
$$

Let $j^{*} \in J^{*}$. If

$$
\sum_{k \in K_{j^{*}}}\left(\sum_{i \in I} c_{i j^{* k}} x_{i j^{* k}}-F_{j^{* k}}\right)-f_{j^{*}}>0
$$

then by the nonnegativity assumption we also have

$$
\sum_{i \in I} \max _{k \in K_{j *}} c_{i j^{* k}}-\sum_{k \in K_{j^{*}}} F_{j^{* k}}-f_{j^{*}} \geq \sum_{k \in K_{j^{*}}}\left(\sum_{i \in I} c_{i j^{*} k} x_{i j^{* k}}-F_{j^{*} k}\right)-f_{j^{*}}>0
$$

This implies that the solution $\left(t^{\prime}, y^{\prime}, x^{\prime}\right)$ given by

$$
\begin{aligned}
& t_{j k} k^{\prime}=\left\{\begin{array}{ll}
1 & \text { if } j=j^{*}, k \in K_{j^{*}} \\
0 & \text { otherwise }
\end{array}, \quad y_{j}{ }^{\prime}= \begin{cases}1 & \text { if } j=j^{*} \\
0 & \text { otherwise }\end{cases} \right. \\
& x_{i, k^{\prime}}= \begin{cases}1 & \text { if } j=j^{*}, k=\operatorname{argmax}_{\prime^{\prime} \in \mathrm{K}_{j^{\prime}} \mathrm{c}_{\mathrm{ij}}{ }^{* \prime}}\end{cases}
\end{aligned}
$$

is feasible with a positive objective value. Since $\vartheta(2 E L)=0$ this contradicts the optimality of $(t, y, x)$ and so for $j^{*} \in J^{*}$ we have

$$
\sum_{k \in K, *}\left(\sum_{i \in I} c_{i j^{* k}} x_{i, j^{*} k}-F_{j^{* k}}\right)-f_{j^{*}}=0
$$

As in the proof of Lemma 3.1, it follows, for any $j^{*} \in J^{*}$, that the solution given by (18) solves $(2 E L)$, which concludes the proof. 
Using Lemma 4.1 it is possible by a similar reasoning as in Proposition 3.2 to partially characterize an optimal solution of $(F 2 E L)$.

Proposition 4.2. If $c_{i j k} \geq 0$ for all $i \in I, j \in J, k \in K$ and $f_{j}, F_{j k}>0$ for all $j \in J, k \in K$ then there exists an optimal solution of (F2FI.) with only one facility open.

The above proposition shows that instead of solving $(F 2 E L)$ directly we can decompose the original problem into $p$ fractional location problems of the form

$$
\begin{gathered}
\max \frac{\sum_{i \in I} \sum_{k \in K} c_{i j k} x_{i j k}}{\sum_{k \in K} F_{j k} t_{j k}+f_{j}} \\
\text { s.t.: } \sum_{k \in K} x_{i j k}=I \forall i \in I \\
x_{i, j k} \leq t_{j k} \forall i \in I, k \in K \\
t_{j k} \in\{0,1\} \forall k \in K \\
x_{i j k} \geq 0 \forall i \in I, k \in K
\end{gathered}
$$

Observe that the above problem corresponds to $(F F L c)$ and therefore we cannot immediately exhibit an optimal solution. IIowever, if $f_{j}=0$, this problem reduces to an instance of $(F F L)$ and, by Proposition 3.2, it can be easily solved.

Proposition 4.2 shows that instead of solving $(F 2 E L)$ directly, we can decompose this problem into $p$ one-level fractional location problems. Unfortunately, this type of result does not hold for both the general model proposed by Barros and Labbé (1994) and its simplification where no operating costs exist, i.e. the simple two-level location problem; see, for example, Tcha and Lee (1984).

Finally, it is important to note that if no pre-existing contracts with the clients exist, then constraints (13) can be replaced by

$$
\sum_{j \in J} \sum_{k \in K} x_{i, j k} \leq 1 \forall i \in I
$$

yielding the following two-level location problem

$$
\begin{aligned}
& \max \frac{\sum_{i \in I} \sum_{j \in J} \sum_{k \in K} c_{i j k} x_{i j k}}{\sum_{j \in J} f_{j} y_{j}+\sum_{j \in J} \sum_{k \in K} F_{j k} t_{j k}} \\
& \text { s.t.: (23),(14),(15),(16),(17) }
\end{aligned}
$$

Similar to the one-level fractional location problem $(F R F L)$, the above fractional problem is no longer well defined. However, the reasoning used to discuss the one-level case can be adapted as follows. Clearly, if it is decided that an investment is made, and hence at least one pair facility $j$ and depot $k$ is opened, then this problem is equivalent to

$$
\begin{array}{r}
\max \frac{\sum_{i \in I} \sum_{j \in J} \sum_{k \in K} c_{i j k} x_{i j k}}{\sum_{J \in J} f_{i} y_{j}+\sum_{j \in J} \sum_{k \in K} F_{i k} t_{j k}} \\
\text { s.t.: } \sum_{j \in J} \sum_{k \in K} t_{j k} \geq 1
\end{array}
$$




$$
\text { (23),(14),(15),(16),(17) }
$$

Observe that the above problem is a well defined fractional programming problem. Hence, a similar analysis as done for Proposition 3.7, by means of Lemma 3.6, leads to the following result.

Proposition 4.3. If there exists a parameter $c_{i j k}$ positive and $f_{j}, F_{j k}>0$ for all $j \in J, k \in K$ then there exists an optimal solution of (FR2ELO), and also (FR2EL), with only one facility open.

Notice that the above result implies that (FR2ELO) can be decomposed into $p$ one-level fractional location problems of the form

$$
\begin{aligned}
\max \frac{\sum_{i \in I} \sum_{k \in K} c_{i j k} x_{i, k}}{\sum_{k \in K} F_{j k} t_{j k}+f_{j}} \\
\text { s.t.: } \sum_{k \in K} t_{j k} \geq 1
\end{aligned}
$$$$
\text { (19),(20),(21),(22) }
$$

Acknowledgements-We would like to thank the referees for their comments.

\section{REFERENCES}

Barros, A. I. (1995) Discrete and Fractional Programming Techniques for Location Models, Tinbergen Institute Research Series, 89, Thesis Publishers, Amsterdam.

Barros, A. I. \& Labbé, M. (1994) A general model for the uncapacitated facility and depot location problem. Location Science, 2, 173-191.

Brealey, R. A. and Myers, S. C. (1988) Principles of Corporate Finance, McGraw-Hill, Singapore.

Cornuéjols, G., Nemhauser, G. L. and Wolsey, L. A. (1990) The uncapacitated facility location problem, In Discrete Location Theory, eds. P. B. Mirchandani and R. L. Francis, Ch. 3, Wiley-Interscience, New York.

Dinkelbach, W. (1967) On nonlinear fractional programming. Management Science, 13, 492-498.

Gao, L. L. \& Robinson, E. P. Jr (1992) A dual-based optimization procedure for the two-echelon uncapacitated facility location problem. Naval Research Logistics, 39, 191-212.

Hansen, P., Pedrosa Filho, E. L. \& Ribeiro, C. C. (1994) Modelling location and sizing of offshore platforms. European Journal of Operational Research, 72, 602-605.

Hansen, P., Poggi de Aragão, M. V. \& Kibeiro, C. C. (1991) Hyperbolic 0-1 programming and query optimization in information retrieval. Mathematical Programming, 52, 255-263.

Hashizume, S., Fukushima, M., Katoh, N. \& Ibaraki, T. (1987) Approximation algorithms for combinatorial fractional programming problems. Mathematical Programming, 37, 255-267.

Meggido, N. (1979) Combinatorial optimization with rational objective functions. Mathematics of Operations Research, 4, 414-424.

Revelle, C. \& I aporte, G. (1993) New directions in plant Incation. Studies in I.ocational Analysis, $5,31-58$.

Schaible, S. (1995) Fractional programming, In Handbook of Global Optimization, eds. R. Horst and P. M. Pardalos, Kluwer, Dordrecht.

Tcha, D. \& Lee, B. (1984) A branch-and-bound algorithm for the multi-level uncapacitated facility location problem. European Journal of Operational Research, 18, 35-43. 